\title{
Caractérisation physico-chimique des eaux usées d'abattoir en vue de la mise en œuvre d'un traitement adéquat : cas de Kénitra au Maroc
}

\section{Driss BELGHYTI ${ }^{*}$, Youssef El GUAMRI ${ }^{\prime}$, Ghizlane ZTIT' ${ }^{1}$, My. Lahcen OUAHIDI ${ }^{2}$, My Brahim JOTI ${ }^{3}$, Abdelatif HARCHRASS ${ }^{1}$, Hammou AMGHAR ${ }^{2}$, Ouafae BOUCHOUATA ${ }^{4}$, Khadija El KHARRIM ${ }^{1}$ et Hamid BOUNOUIRA ${ }^{5}$}

'Equipe Environnement et Parasitologie. Laboratoire de Biologie \& Santé. / Projet SVS 18/99. UFR Doctorale : Parasitologie Comparée : Applications Médicales et Vétérinaires. Faculté des Sciences de Kénitra / Université Ibn Tofail. B.P.: 133 / 14000 Kénitra (Maroc).

${ }^{2}$ Laboratoire National de L'Environnement. Ministère de l'Energie, des Mines, de l'Eau et de l'Environnement. Rabat. Maroc.

${ }^{3}$ Division de l'Assainissement Liquide. Office National de l'Eau Potable (ONEP). Kénitra. Maroc.

${ }^{4}$ Autorité Nationale Désignée - Service Coopération Multilatérale. Direction du

Partenariat, de la Communication et de la Coopération. Département de

l'Environnement. Ministère de l'Energie, des Mines, de l'Eau et de l'Environnement. Rabat. Maroc.

${ }^{5}$ Unité Géochimie et Pollution (U.G.P). Département d'Applications aux Sciences de la Terre et de I'Environnement (D.A.S.T.E). Centre National d'Energie, des Sciences et des Techniques Nucléaires (CNESTEN) B.P. 1382 R.P. 10001-Rabat-Maroc.

*Correspondance,courriel : belghyti@univ-ibntofail.ac.ma/belghyti@hotmail.com.

\section{Résumé}

Notre objectif principal est de caractériser les eaux usées d'abattoir municipal de la ville de Kénitra (Maroc) et de recommander un traitement adéquat permettant leurs réutilisations, réduisant ainsi les nuisances que subit le milieu récepteur (Oued Sebou) et de remédier aussi à la perte de cette source hydrique en matières valorisables. La caractérisation physico-chimique des eaux usées brutes a révélé que ce rejet liquide est très chargé en matière organique en terme de $D C O$ (Moy. $=$

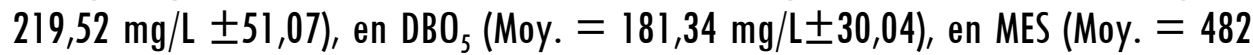


$\mathrm{mg} / \mathrm{L} \pm 222,7)$ et en matière minérale exprimée en terme de Chlorures (Moy. $=237$ $\mathrm{mg} / \mathrm{L} \pm 109,6$ ), en Conductivité électrique (Moy. $=1360,5 \mu \mathrm{s} / \mathrm{cm} \pm 1120,8$ ) et en Alcalinité exprimée en $\mathrm{CaCO}$ (Moy. $=201 \mathrm{mg} / \mathrm{L} \pm 74,2$ ) avec un pH de 7,5 ( $\pm 0,8)$. Les teneurs moyennes en Nitrates et en Orthophosphates respectivement de l'ordre de $1,74 \mathrm{mg} / \mathrm{L}( \pm 2,7)$ et $0,08 \mathrm{mg} / \mathrm{L}( \pm 0,1)$. Malgré que ces eaux usées présentent une charge organique élevée (rapports $\mathrm{DBO}_{5} / \mathrm{DCO}=0,83$ et $\mathrm{MES} / \mathrm{DBO}_{5}=2,66$ ), elles présentent une biodégradabilité satisfaisante. L'examen de rapport $\mathrm{DCO} / \mathrm{DBO}_{5}=$ 1,21 souligne bien le caractère biodégradable des eaux usées de l'abattoir municipal de Kénitra auxquelles un traitement biologique paraît tout à fait convenable. Cependant, il faut tenir compte les eaux usées domestiques mélangées avec les effluents de l'abattoir. Il existe une corrélation hautement significative entre la $D C O$ et la $\mathrm{DBO}_{5}$ de nos échantillons d'eaux usées $\left(\mathbb{R}^{2}=0,93\right.$ et $\left.y=1,2689 x+23,814\right)$. Par ailleurs, la corrélation établie entre l'oxygène dissous et la DB05 montre un $R^{2}=$ $0,75(y=0,0057 x+0,7964)$.

Mots-clés : eaux usées, abattoir, paramètres physico-chimiques, traitement, Kénitra.

\section{Abstract \\ Physicochemical study of wastewater of municipal slaughter-house for the implementation of a suitable treatment (Kenitra city -Morocco)}

The goal of our study is to characterize the effluent of the municipal slaughter-house of the Kenitra city and to recommend a suitable treatment allowing it's recycling, thus reducing the harmful effect which undergoes the receiving environment (Sebou) and to also mitigate the loss of this hydrous source out of matters which may undergo beneficiation. The physicochemical characterization of raw wastewater revealed that this liquid rejection is very charged out of organic matter expressed in term with COD (Avg.= $199.52 \mathrm{mg} / \mathrm{L} \pm 51.07)$, in $\mathrm{BDO}_{5}(\mathrm{Avg} .=241.34 \mathrm{mg} / \mathrm{L} \pm 30.04)$, in Suspend Matter (Avg. $=482 \mathrm{mg} / \mathrm{L} \pm 222.7$ ) and out of mineral matter expressed in term of Chlorides (Avg. $=237 \mathrm{mg} / \mathrm{L} \pm 109.6)$, in Electric Conductivity (Avg. $=1360.5$ $\mu \mathrm{s} / \mathrm{cm} \pm 1120,8)$ and in Alkalinity expressed in $\mathrm{CaCO}_{3}(\mathrm{Avg} .=201 \mathrm{mg} / \mathrm{KL} \pm 74.2)$ with a pH of $7.5( \pm 0.8)$. The average content of Nitrates and Orthophosphates are about $1.74 \mathrm{mg} / \mathrm{L}( \pm 2.7)$ and $0.08 \mathrm{mg} / \mathrm{L}( \pm 0.1)$. Although this wastewater presents a high organic load (reports $\mathrm{BDO}_{5} / \mathrm{COD}=0.83$ and $\mathrm{SM} / \mathrm{BDO}_{5}=2.66$ ), they have a satisfactory biodeterioration. The examination of report $\mathrm{COD} / \mathrm{BDO}_{5}=1.21$ underlines well the biodegradable character of mixed wastewater of the municipal slaughterhouse of Kenitra city to which a biological treatment appears completely suitable. 
However, it should be sufficient to hold account the domestic wastewater mixed with the effluents of the slaughterhouse. There is a highly significant correlation between $\mathrm{COD}$ and $\mathrm{BOD}_{5}(\mathrm{R}=0.92)$. The correlation between dissolved oxygen and $\mathrm{BOD}_{5}$ showed a R2 $=0.80$.

Keywords : Slaughter-house wastewaters, characterization, treatment, Kenitra.

\section{Introduction}

L'eau est un des éléments essentiels de la plupart des grandes entreprises de transformation de produits alimentaires d'origine animale. Après avoir été utilisée, la plus grande partie de cette eau usée de procédé est retournée à l'environnement. Comme cette eau est habituellement chargée en matière organique, elle devient dès lors une source de pollution importante pour le milieu récepteur qui la reçoit.

Les abattoirs constituent sans doute l'exemple-type de ces industries où l'eau est utilisée pour le lavage des sous-produits (abats) et l'élimination des déchets (matières fécales, débris de panse et de sang). En Europe, les volumes d'eau usée rejetés sont évalués entre 6 et 9 litres par $\mathrm{kg}$ de carcasse de bovins, et de 5 à 11 litres par $\mathrm{Kg}$ de carcasse de porcins [1]. Les effluents, de ces abattoirs sont caractéristiques et nécessitent des traitements adaptés (séparation des déchets solides et des graisses, traitements spécifiques). Plusieurs études se sont intéressées à la caractérisation et au traitement de ce type d'eaux usées par le biais des stations d'épuration soit par des procédés aérobies [2,3]. Soit par de procédés anaérobies [4-7]. D'autres procédés de traitement sont adoptés pour l'épuration des eaux usées d'abattoir à savoir l'infiltration sur sable [8] et l'électrocoagulation [9].

La plupart des données sur la qualité des eaux usées d'abattoir ont été produites en Europe [10-13], Australie [14], les Etats-Unis [15] et l'Afrique [16-18] et peu d'information existe sur la caractérisation et le traitement des eaux usées d'abattoir au Maroc.

La capacité nominale de l'abattoir municipal de la ville de Kénitra (Maroc) est de 3500 tonnes de carcasses abattues par an correspondant à 560 tonnes de déchets. Vu sa proximité à l'océan atlantique (Mehdia), les effluents de cet abattoir sont rejetés directement dans l'oued Sebou sans traitement préalable. Ces eaux usées constituent une source de pollution pour les eaux de surface et l'océan. Elles peuvent être responsable d'un déséquilibre écologique irréversible ainsi que de l'eutrophisation des eaux de milieu récepteur. Comme ce dernier rejetait ses eaux usées dans le collecteur urbain $(C)$, le traitement de cet effluent est fortement recommandé dans le 
contexte global d'assainissement des eaux usées de la ville de Kénitra par la recherche d'un traitement convenable. Le présent travail consiste d'abord a évaluer les effluents de l'abattoir municipal de Kénitra, à faire ensuite une caractérisation physico-chimique par la détermination de certains paramètres majeurs et globaux de la pollution des eaux usées et enfin de décrire les mesures de protection du milieu récepteur par la recherche d'un moyen de traitement convenable de ces effluents et par la mise en place d'une station de traitement.

\section{Matériel et méthodes}

\section{2-1. Site d'étude}

La ville de Kénitra est située à $40 \mathrm{Km}$ au Nord de la capitale du Maroc, entre les méridiens $6^{\circ} 30^{\prime}$ et $6^{\circ} 45^{\prime}$ Ouest et les parallèles $34^{\circ} 15^{\prime}$ et $34^{\circ} 20^{\prime}$. Elle est dotée d'un réseau d'assainissement de type unitaire qui dessert environ $78 \%$ de la population. Le reste utilise des systèmes d'assainissement individuel par puits perdus ou fosses septiques. En 2004, la production de viandes rouges est de 282752 tonnes dont 50\% $d^{\prime}$ origine bovine, $42 \%$ d'origine ovine et $8 \%$ d'origine caprine. En ce qui concerne les viandes bovines, 143246 tonnes soit $90 \%$ sont inspectées au niveau de l'abattoir [19]. Les eaux usées de ce rejet sont chargées par les déchets solides, des matières graisses, l'abondance de la matière organique (débris de panse) et sont concentrées par le sang des animaux abattus [20].

\section{2-2. Prélèvement et analyses des eaux usées}

Les paramètres physico-chimiques sont déterminés à partir de prélèvements bimensuels (entre Février et Juin 2005) effectués au niveau de l'exutoire de rejet d'abattoir municipal de Kénitra. La conservation des prélèvements d'eaux usées a été faite selon le guide général pour la conservation et la manipulation des échantillons d'après ISO 5667/3 [21].

Le $\mathrm{pH}$, la température, la conductivité électrique, l'oxygène dissous sont déterminés à l'aide d'un un multi-parameter analyser Type CONSORT - Modèle 835. La DBO 5 est déterminée par la méthode respiratoire à l'aide d'un DBO-mètre marque WTW, modèle 1020 T selon la technique décrite par DIN [22]. La DCO est déterminé par l'oxydation en milieu acide par l'excès de dichromate de potassium à la température de $148^{\circ} \mathrm{C}$ des matières oxydables dans les conditions de l'essai en présence de sulfate d'argent comme catalyseur et de sulfate de mercure [23]. Les chlorures et l'alcalinité exprimée en $\mathrm{CaCO}$ sont déterminés par la méthode de comptage des 
pastilles prédosées. Les matières en suspension sont déterminées par filtration d'un volume d'eau usée sur filtre cellulosiques (de $0,45 \mu \mathrm{m}$ ) selon Rodier [24]. Les Orthophosphates sont déterminés par la méthode colorimétrique par complexe phosphomolybdique selon DIN [25]. Les Nitrates sont dosés par la méthode photométrique avec 2,6 - diméthylphénol selon DIN [26] et enfin la détermination des Nitrites a été effectuée par la méthode photométrique selon DIN [27].

\section{Résultats}

L'évaluation de la pollution d'une eau usée brute se fait d'après la détermination d'un certain nombre de paramètres physico-chimiques caractérisant cette eau usée. Les caractéristiques physico-chimiques des eaux usées de l'abattoir sont regroupées dans le Tableau 1 .

Tableau 1 : Paramètres physico-chimiques des eaux usées de l'abattoir de Kénitra.

\begin{tabular}{|c|c|c|c|c|c|}
\hline Paramètres & Moy. & Max. & Min. & Ecart-type & $\begin{array}{c}\text { Nombre de } \\
\text { prélèvement }\end{array}$ \\
\hline $\mathrm{T}^{\circ} \mathrm{C}$ & 21,5 & 26 & 16,8 & 6,5 & 10 \\
\hline $\mathrm{pH}$ & 7,5 & 8,2 & 7,1 & 0,8 & 10 \\
\hline C.E $(\mu \mathrm{s} / \mathrm{cm})$ & 1360,5 & 1945 & 360 & 1120,8 & 10 \\
\hline $\begin{array}{l}\text { Alcalinité } \\
\left.\mathrm{CaCO}_{3}\right)\end{array} \quad(\mathrm{mg} / \mathrm{L} \quad$ de & 201 & 240 & 135 & 74,2 & 10 \\
\hline Oxygène dissous (mg/L) & 1,8 & 2,42 & 1,05 & 1,0 & 10 \\
\hline MES (mg/L) & 482 & 670 & 355 & 222,7 & 10 \\
\hline $\mathrm{DBO}_{5}(\mathrm{mg} / \mathrm{L})$ & 181,34 & 220,5 & 30,04 & 136,52 & 10 \\
\hline$D C O(\mathrm{mg} / \mathrm{L})$ & 219,52 & 307,2 & 51,07 & 101,65 & 10 \\
\hline Nitrates (mg/L) & 1,742 & 4,125 & 0,349 & 2,7 & 10 \\
\hline Nitrites (mg/L) & 0,029 & 0,036 & 0,023 & 0,0 & 10 \\
\hline $\mathrm{PO}_{4}{ }^{3 .}(\mathrm{mg} / \mathrm{L})$ & 0,089 & 0,147 & 0,059 & 0,1 & 10 \\
\hline Chlorures (mg/L) & 237 & 315 & 160 & 109,6 & 10 \\
\hline
\end{tabular}

La température des eaux usées du collecteur urbain (C), qui draine l'abattoir municipal et la nouvelle Médina (Kénitra), est comprise entre $16.8^{\circ} \mathrm{C}$ et $26^{\circ} \mathrm{C}$ comme valeurs extrêmes minimales et maximales et $21.5^{\circ} \mathrm{C}$ comme valeur moyenne (Tableau I). D'une manière générale, les échantillons des eaux usées analysées ont 
un pH relativement neutre. Pour la conductivité électrique, les valeurs moyennes enregistrées se situent entre $360 \mu \mathrm{s} / \mathrm{cm}$ et $1945 \mu \mathrm{s} / \mathrm{cm}$ avec $1360,5 \mu \mathrm{s} / \mathrm{cm}$ comme valeur moyenne (Tableau ). Les valeurs de Chlorures dans les eaux usées se situent entre $160 \mathrm{mg} / \mathrm{L}$ et $315 \mathrm{mg} / \mathrm{L}$ avec $237 \mathrm{mg} / \mathrm{L}$ comme valeur moyenne (Tableau ク). Alors que, les valeurs trouvées de l'alcalinité montrent que les eaux usées de ce collecteur sont caractérisées par une concentration moyenne en bicarbonates de calcium $\left(\mathrm{CaCO}_{3}\right)$ de l'ordre de $201 \mathrm{mg} / \mathrm{L}$ ( Tableav $)$. Les Nitrates comme les autres formes azotés évoluent très rapidement dans le milieu naturel selon le cycle d'azote. Les valeurs de Nitrates enregistrées au niveau des rejets du collecteur (C) présentent des variations considérables pendant la période d'étude. Les teneurs en Nitrates des effluents de l'abattoir municipal varient entre $0,349 \mathrm{mg} / \mathrm{L}$ et $4,125 \mathrm{mg} / \mathrm{L}$ avec une concentration moyenne de $1,742 \mathrm{mg} / \mathrm{L}$. En revanche, les valeurs de Nitrites enregistrées dans les rejets d'abattoir ne présentent pas des variations considérables. Les teneurs en Nitrites des eaux usées analysées varient entre $0,023 \mathrm{mg} / \mathrm{L}$ et $0,036 \mathrm{mg} / \mathrm{L}$ avec une valeur moyenne de l'ordre de $0,029 \mathrm{mg} / \mathrm{L}$ (Tableau 1). De même, les teneurs enregistrées en orthophosphates ne présentent pas de variations considérables au cours du cycle de prélèvement effectué. Les valeurs enregistrées varient entre $0,059 \mathrm{mg} / \mathrm{L}$ et $0,147 \mathrm{mg} / \mathrm{L}$ avec une valeur moyenne de $0,089 \mathrm{mg} / \mathrm{L}$. L'évolution mensuelle des concentrations en orthophosphates dans le rejet a montré que ces effluents bruts sont moins concentrés. (Tableau I).

Les concentrations en MES des eaux usées analysées varient entre $355 \mathrm{mg} / \mathrm{L}$ et $670 \mathrm{mg} / \mathrm{l}$ avec une moyenne de $482 \mathrm{mg} / \mathrm{L}$ (Tableau ). L'état d'oxygénation de ces rejets montre que, les valeurs extrêmes minimales et maximales de la teneur en oxygène dissous sont de $1,05 \mathrm{mg} / \mathrm{L}$ et $2,42 \mathrm{mg} / \mathrm{L}$ avec une concentration moyenne de $1,8 \mathrm{mg} / \mathrm{L}$ (Tableau $)$. Les valeurs de pollution organique exprimée en $\mathrm{DBO}_{5}$ présentent des variations négligeables entre les différentes campagnes de prélèvements. Les valeurs de $\mathrm{DBO}_{5}$ enregistrées varient entre $30,04 \mathrm{mg} / \mathrm{L}$ (valeur minimale) et $220,5 \mathrm{mg} / \mathrm{L}$ (valeur maximale) avec une valeur moyenne de $181,34 \mathrm{mg} / \mathrm{L}$ (Tableau $)$. Les valeurs de la DCO présentent une variation non négligeable au cours de la période d'étude. Les valeurs varient entre $51,07 \mathrm{mg} / \mathrm{L}$ et $307,2 \mathrm{mg} / \mathrm{L}$ avec une moyenne de 219,52 mg/L (Tableau $)$. 


\section{Discussion}

\section{4-1. Paramètres physico-chimiques des eaux usées mélangées d'abattoir}

Les valeurs de la température des eaux usées enregistrées sont inférieures à $30^{\circ} \mathrm{C}$ considérée comme valeur limite de rejet direct dans le milieu récepteur [28]. De même, ces valeurs sont inférieures à $35^{\circ} \mathrm{C}$, considérée comme valeur limite indicative pour les eaux destinées à l'irrigation [28] (Tableau 2).

Le pH, indique l'alcalinité des eaux usées, son rôle est capital pour la croissance des microorganismes qui ont généralement un pH optimum variant de 6,5 à 7,5. Lorsque le $\mathrm{pH}$ est inférieur à 5 ou supérieur à 8,5, la croissance des microorganismes est directement affectée. En outre, le pH est un élément important pour l'interprétation de la corrosion dans les canalisations des installations de l'épuration. Les valeurs de pH mesurés varient peu et restent autour de 7,5 en moyenne. Les valeurs obtenues sont comparables à celles trouvées ailleurs pour les eaux usées d'abattoirs qui présentent généralement un pH neutre à légèrement basique [29-31,7]. En revanche, elles ne sont par en accord avec ceux trouvées par Wéthé et al, [16] au niveau des eaux usées d'abattoir de Burkina Faso.

La conductivité électrique est probablement l'une des plus simple et des plus importantes pour le contrôle de la qualité des eaux usées. Elle traduit le degré de minéralisation globale, elle nous renseigne sur le taux de salinité. C'est une expression numérique de la capacité de l'eau à conduire un courant électrique mesurée en millisiemens par centimètre. Les résultats obtenus mettent en évidence une variation plus ou moins importante de la minéralisation exprimée en conductivité moyenne. Ces résultats pourraient être expliqué d'une part par le rejet des eaux usées résiduaires riches en fertilisants artificiels d'une industrie d'engrais (NPK) et d'autre part au rejet des déchets d'abattoir municipal fortement minéralisés raccordé à ce collecteur. Nisbet [32] a signalé que des valeurs moyennes, comprises entre $449,7 \mu \mathrm{s} / \mathrm{cm}$ et $1037,3 \mu \mathrm{s} / \mathrm{cm}$, mettent en évidence une forte minéralisation des eaux usées. La comparaison des valeurs de la conductivité électrique au niveau des eaux usées analysées avec les normes de qualité des eaux destinées à l'irrigation permet de déduire que ces eaux usées sont acceptables pour l'irrigation des cultures. De même, ces valeurs moyennes sont inférieures à $2700 \mu \mathrm{s} / \mathrm{cm}$, considérée comme valeur limite de rejet direct dans le milieu récepteur [28] (Tableau 2). 
Tableau 2 : Valeurs limites pour les différents rejets que se soit directs (eaux pluviales), indirects (eaux usées) ou les eaux destinées à l'irrigation des cultures. [28].

\begin{tabular}{|c|c|c|c|}
\hline Paramètres & VLR rejet diredt & VLR rejet indired & $\begin{array}{l}\text { Eaux destinées ò } \\
\text { l'irrigation }\end{array}$ \\
\hline Température & $30^{\circ} \mathrm{C}$ & $35^{\circ} \mathrm{C}$ & $35^{\circ} \mathrm{C}$ \\
\hline $\mathrm{pH}$ & $6,5-0,51$ & $6,5-8,51$ & $6,5-85$ \\
\hline $\mathrm{DBO}_{5}$ & $100 \mathrm{mg} / \mathrm{l}$ & $500 \mathrm{mg} / \mathrm{l}$ & -- \\
\hline $\mathrm{DCO}$ & $500 \mathrm{mg} / \mathrm{l}$ & $1000 \mathrm{mg} / \mathrm{I}$ & -- \\
\hline MES & $50 \mathrm{mg} / \mathrm{l}$ & $600 \mathrm{mg} / \mathrm{l}$ & $2000 \mathrm{mg} / \mathrm{l}$ \\
\hline Conductivité & $2700 \mu \mathrm{s} / \mathrm{cm}$ & & $8,7 \mathrm{~ms} / \mathrm{cm}$ \\
\hline $\begin{array}{lr}\text { Bicarbonate } & \left(\mathrm{HCO}_{3}\right) \\
\text { Irrigation } & \text { par } \\
\text { aspersion } & \end{array}$ & -- & -- & $518 \mathrm{mg} / \mathrm{l}$ \\
\hline Sulfates & -- & 400 & $250 \mathrm{mg} / \mathrm{l}$ \\
\hline Anote Kjeldahl & $30 \mathrm{mgl} / \mathrm{l}$ & & \\
\hline Phosphore total & $10 \mathrm{mgP} / \mathrm{l}$ & $10 \mathrm{mgP} / \mathrm{I}$ & \\
\hline Chlorure (C) & $\begin{array}{c}\text { Chlor e adif (I) } 20,2 \\
\mathrm{mg} / \mathrm{l})\end{array}$ & -- & $\begin{array}{l}\text { Irrigation de surface (350 } \\
\mathrm{mg} / \mathrm{l})\end{array}$ \\
\hline & & & $\begin{array}{c}\text { Irrigation par aspersion } \\
(105 \mathrm{mg} / \mathrm{l})\end{array}$ \\
\hline $\mathrm{H}-\mathrm{HO}_{3}$ & & & $50 \mathrm{mg} / \mathrm{l}$ \\
\hline
\end{tabular}

Les variations de l'alcalinité des eaux usées sont rapprochées de celles du degré de minéralisation (conductivité électrique, dureté totale, pH) qui donne aussi une indication sur le degré d'oxydation des composés organiques (cas des eaux résiduaires). La concentration des carbonates $\left(\mathrm{CO}_{3}{ }^{2-}\right)$ et des bicarbonates $\left(\mathrm{HCO}_{3}\right)$ dans l'eau est fonction de la teneur en $\mathrm{CO}_{2}$ car ce dernier est très soluble dans l'eau (200 
fois plus que l'oxygène) et sa solubilité dépend de la température et la pression atmosphérique. La concentration légèrement élevée de $\mathrm{CaCO}_{3}$ au niveau des échantillons analysés pourrait être attribuée aux rejets des eaux usées moins chargées en matières organiques fermentescibles susceptibles d'être oxydées et ayant pour conséquence, une production élevée de $\mathrm{CO}_{2}[33]$. Le $\mathrm{CaCO}_{3}$ peu soluble dans l'eau; cependant, la présence du $\mathrm{CO}_{2}$ confère à l'eau une force de dissolution bien plus importante en transformant le Carbonate de calcium en bicarbonate de calcium, lesquels sont plus solubles dans l'eau selon la réaction suivante:

$$
\mathrm{CaCO}_{3}+\mathrm{CO}_{2} \longrightarrow \mathrm{Ca}\left(\mathrm{HCO}_{3}\right)_{2} \longrightarrow \mathrm{Ca}^{++}+2 \mathrm{HCO}^{3-}
$$

Au niveau des effluents d'abattoirs de Canada, Massé et Masse, [5] ont rapportés des valeurs moyennes entre 83 et $900 \mathrm{mg} / \mathrm{L}$ en $\mathrm{CaCO}_{3}$. Les mêmes auteurs ont signalés des valeurs très élevées entre 667 et $1056 \mathrm{mg} / \mathrm{L}$ dans une autre étude au Canada [5]. Les normes marocaines [28] recommandent une concentration en $\mathrm{HCO}_{3}$ - de $250 \mathrm{mg} / \mathrm{L}$ pour les eaux destinées à l'irrigation des cultures (Tableau 2). Les valeurs de la conductivité indiquent une forte minéralisation de ces eaux usées pendant toute la période de prélèvement et permettent de constater que les variations temporelles de ce paramètre sont plus importantes.

L'azote présent dans l'eau usée peut avoir un caractère organique ou minéral. L'azote organique est principalement un constituant des protéines, des polypeptides, des acides aminés et de l'urée. L'azote minéral qui comprend l'ammonium $\left(\mathrm{NH}_{4}{ }^{+}\right.$), les Nitrites $\left(\mathrm{NO}_{2}\right)$ et les Nitrates $\left(\mathrm{NO}_{3}{ }^{-}\right)$constitue la majeure partie de l'azote total. Des valeurs très élevées de l'azote total (Nt) ont été enregistrés dans les eaux usées d'abattoirs du Brésil (entre 133 et $179 \mathrm{~g} / \mathrm{l}$ ) par Miranda et al., [31] et une teneur moyenne en $\mathrm{NH}_{4}^{+}$de 163,7 mg/L [7]. Qu'au niveau des eaux usées d'abattoir d'El Jadida (Maroc), Chennaoui et al., [31] ont rapportés une teneur moyenne de $12 \mathrm{~g} / \mathrm{L}$.

Les teneurs en Nitrates dans les effluents de l'abattoir de Kénitra varient entre $0,349 \mathrm{mg} / \mathrm{L}$ ef $4,125 \mathrm{mg} / \mathrm{L}$ avec une concentration moyenne de $1,742 \mathrm{mg} / \mathrm{L}$ L'accroissement de leur teneur peut provenir des effluents riches en NPK et en éléments fertilisants d'une usine d'engrais raccordée à ce collecteur et en déchets organiques de l'abattoir. Les teneurs restent dans la majorité des cas inférieurs à $2 \mathrm{mg} / \mathrm{L}$ à l'exception d'un pic observé au mois d'avril ou il y a de fortes pluies. Ces résultats sont comparables à ceux observés par Khamar [34] et Zerhouni [35]. Pour les Nitrites, qui constituent une étape importante dans la métabolisation des composés azotés, ils s'insèrent aussi dans le cycle d'azote entre l'ammonium et les nitrates. Les Nitrites proviennent généralement soit d'une dégradation incomplète d'Ammoniac soit d'une réduction des Nitrates, ils ne représentent qu'un stade 
intermédiaire et facilement oxydés en nitrates (par voie chimique ou bactérienne). Les faibles concentrations en Nitrites rencontrées au niveau des eaux usées de l'effluent étudié, pourraient être expliquer par le fait que l'ion Nitrite $\left(\mathrm{NO}_{2}{ }^{-}\right)$est un composé intermédiaire, instable en présence de l'oxygène, dont la concentration est généralement très inférieure à celle des deux formes qui lui sont liées, les ions nitrates et ammonium [33]. La comparaison des concentrations moyennes en Nitrates dans les eaux usées analysées avec la norme de qualité des eaux destinées à l'irrigation montre que, ces concentrations sont inférieures à $50 \mathrm{mg} / \mathrm{L}$, ce qui permet de déduire que ces effluents sont acceptables pour l'irrigation des cultures [28] (Tableau 2).

Les composés phosphorés existent dans les eaux naturelles ef les eaux usées sous différentes formes à savoir les orthophosphates solubles, les phosphates hydrosolubles et les dérivés organophosphorés [24]. Les teneurs enregistrées en orthophosphates ne présentent pas de variations considérables au cours du cycle de prélèvement effectué. Les valeurs enregistrées varient entre $0,059 \mathrm{mg} / \mathrm{L}$ et $0,147 \mathrm{mg} / \mathrm{L}$ avec une valeur moyenne de $0.089 \mathrm{mg} / \mathrm{L}$. Chennaoui et al., [31] ont rapporté une teneur moyenne en Orthophosphate de l'ordre de 1,8g/L. Au niveau des eaux usées d'abattoir de Canada, Massé et Masse, [29] ont trouvé des valeurs comprises entre 25 et $42 \mathrm{mg} / \mathrm{L}$.

D'une manière générale, la MES intervient dans la composition de l'eau par son effet d'échanges d'ions ou d'absorption aussi bien sur les éléments chimiques à l'état de traces que sur les microorganismes. Les variations mensuelles de ces deux paramètres sont indiquées dans la figure 6. D'après les résultats, on note que les eaux usées de l'abattoir ont les mêmes charges que les eaux usées urbaines marocaines pour lesquelles les concentrations en MES se situent entre 250 et $700 \mathrm{mg} / \mathrm{L}$ [36]. Par ailleurs, ces valeurs moyennes en MES dans les eaux usées analysées sont supérieures à la valeur fixée par les normes marocaines [28] (Tableau 2). Ces valeurs trouvées sont moins élevées par rapport aux résultats trouvés par Massé et Masse au Canada [5]. Par ailleurs, Chennaoui et al., [30] ont signalés une concentration moyenne de $13, \mathrm{lg} / \mathrm{L}$.

La signification de paramètre de l'oxygénation des eaux est très claire puisque la présence d'oxygène dissous conditionne les réactions de dégradation -aérobie- de la matière organique et plus généralement l'équilibre biologique des milieux hydriques. Dans les réseaux d'assainissement des eaux usées, sa disparition complète s'accompagne généralement de l'apparition $\mathrm{d}^{\prime} \mathrm{H}_{2} \mathrm{~S}$ dans l'air, provenant de la réduction des composés soufrés présents dans les effluents, et corrélativement du phénomène d'attaque acide du béton des canalisations [33]. Par contre sa présence 
inhibe les activités dénitrifiantes de la flore spécialisé. La comparaison des valeurs en oxygène dissous dans nos échantillons d'eaux usées analysées avec la grille de qualité des eaux de surface permet de déduire que ces eaux usées sont de qualité moyenne à très mauvaise [28] (Tableau 2).

Les valeurs élevées de la $\mathrm{DBO}_{5}$, pourraient être expliqué par l'abondance de la matière organique (débris de panse), et par la concentration de cet effluent par le sang des rejets de l'abattoir municipal drainés. Pour la $D C O$, les valeurs sont moins faibles que celles des eaux urbaines marocaines $(500-1500 \mathrm{mg} / \mathrm{L})$ [36]. Ces valeurs moyennes sont inférieures à $500 \mathrm{mg} / \mathrm{L}$, considérée comme valeur limite de rejet direct [28]. Par ailleurs, ces eaux usées sont classées comme de très mauvaises selon les normes de qualité des eaux de surface [28] (Tableav 2).

Les valeurs de la DCO présentent une variation non négligeable au cours de la période d'étude Cette observation est similaire à celle faite par Khamar [34] et Zerhouni [35]. La charge polluante des eaux usées, telle que mesurée par sa DCO, est un des plus importants critères utilisés dans la conception d'un traitement des eaux usées afin de déterminer le degré de traitement nécessaire. La charge à traiter est la DCO des eaux usées qui y sont amenées [37]. En outre, ces valeurs sont moins élevées et ne concordent pas avec celles reportées par Sachon en France [11]; de Tritt et Shuchard en Allemend [13]; de Gnagne et al. au Burkina Faso [8-18] et les travaux de Miranda et al., [31], Reginatto et al. [7] au Brésil.

\section{4-2. Evaluation de la pollution organique des eaux usées}

Pour une meilleure appréciation de l'origine des eaux usées de ces effluents étudiés de l'abattoir municipal et de la nouvelle Médina (Kénitra), le calcul des rapports $\mathrm{DCO} / \mathrm{DBO}_{5}, \mathrm{DBO}_{5} / \mathrm{DCO}, \mathrm{MES} / \mathrm{DBO}_{5}$ et l'estimation de la Matière Oxydable (MO) présente des intérêts très importants (Tableav 3).

Tableau 3 : Rations des eaux usées de l'abattoir de Kénitra

\begin{tabular}{lcccc}
\hline & Moy. & Max. & Min. & Ecart-type \\
DCO/DBO $_{5}$ & 1,21 & 0,63 & 1,70 & 0,8 \\
DBO $_{5}$ /DCO $_{\text {MES/DBO }_{5}}$ & 0,83 & 1,60 & 0,59 & 0,7 \\
Matières Oxydables (mg/L) $^{2,66}$ & 1,37 & 11,82 & 7,4 \\
\hline
\end{tabular}

L'utilisation de ces paramètres de caractérisation constitue un bon moyen pour donner une image du degré de pollution des effluents bruts d'abattoir et aussi pour 
optimiser les paramètres physico-chimiques de ces eaux usées afin de proposer un mode de traitement convenable.

\section{4-2-1. Ratio $\mathrm{DCO}^{\mathrm{DBO}}$}

Le rapport $\mathrm{DCO} / \mathrm{DBO}_{5}$ permet de déduire si les eaux usées rejetées directement dans le milieu récepteur ont des caractéristiques des eaux usées domestiques (rapport $\mathrm{DCO} / \mathrm{DBO}_{5}$ inférieur à 3) [36]. Les résultats de ce rapport constituent une indication de l'importance des matières polluantes peu ou pas biodégradables [24]. Les eaux usées de l'abattoir municipal présentent un ratio $\mathrm{DCO} / \mathrm{DBO}_{5}$ variant de $0,63 \mathrm{mg} / \mathrm{L}$ à 1,70mg/L (Tableau 3) conforme avec celui des eaux usées urbaines à dominance domestique présentant un rapport $\mathrm{DCO} / \mathrm{DBO}_{5}$ inférieur à 3 [36]. Donc, on peut conclure que même si les eaux usées de ce rejet urbain présentent une charge organique élevée, elles sont facilement biodégradables. L'examen de ce rapport souligne bien le caractère biodégradable des eaux usées mélangées de l'abattoir municipal auxquelles un traitement biologique paraît tout à fait convenable. Ces résultats concordent avec celles reportés par Gnagne et Brissaud [18] et Zerhouni [35].

\section{4-2-2. Ratio $\mathrm{DBO}_{5} / \mathrm{DCO}$}

Pour caractériser une pollution industrielle, on considère souvent le rapport $\mathrm{DBO}_{5} / \mathrm{DCO}$, qui donne des indications très intéressantes sur l'origine d'une pollution des eaux usées et ses possibilités de traitement. Pour notre étude, ce rapport est relativement élevé de l'ordre de 0,83 (Tableau 3). C'est le cas général pour les rejets chargés en matière organique. Cette charge organique rend ces eaux usées assez instables, c'est à dire qu'elles évolueront vite vers des formes "digérées" avec le risque de dégagement d'odeurs. En effet, les eaux usées de ce collecteur sont à dominante organique.

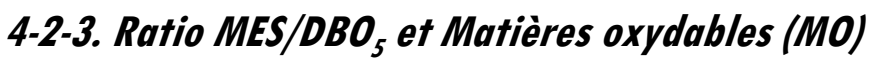

Au niveau des eaux usées de l'abattoir municipal, le rapport $\mathrm{DBO}_{5} / \mathrm{DCO}$ est élevé $(0,83)$, ce qui confirme que les eaux usées drainées par ce collecteur sont fortement chargées en matières organiques (Tableau 3). Ce résultat obtenu est confirmé par l'estimation de la Matière 0xydable, qui est de l'ordre de $227,4 \mathrm{mg} / \mathrm{L}$ avec un rapport moyen de $\mathrm{MES} / \mathrm{DBO}_{5}$ de 2,66. Par ailleurs, le rapport $\mathrm{DCO} / \mathrm{DBO}_{5}$ est faible $(1,2)$, ce qui 
nous permet de déduire que la charge en matières organiques dans les eaux usées de ce collecteur est facilement biodégradable selon Henze et al. [38].

II existe une corrélation hautement significative entre la $\mathrm{DCO}$ et $\mathrm{la} \mathrm{DBO}_{5}$ des eaux usées d'abattoir de la ville de Kénitra.

La figure I montre un $R^{2}=0,93(y=1,2689 x+23,814)$.

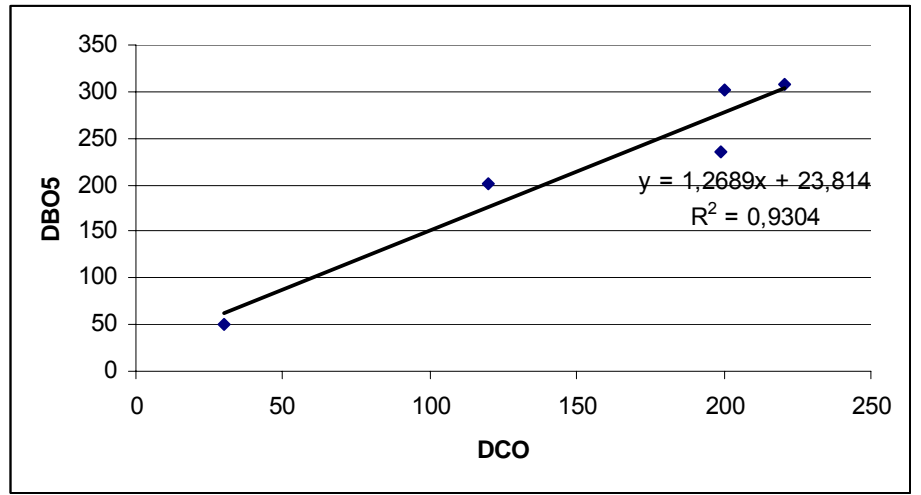

Figure 1: Corrélation établie entre la $\mathrm{DBO}_{5}$ et la DCO des eaux usées du rejet de l'abattoir de Kénitra (Maroc).

Par ailleurs, la corrélation établie entre l'oxygène dissous et la DB05 (Figure 2) montre un $R^{2}=0,75(y=0,0057 x+0,7964)$.

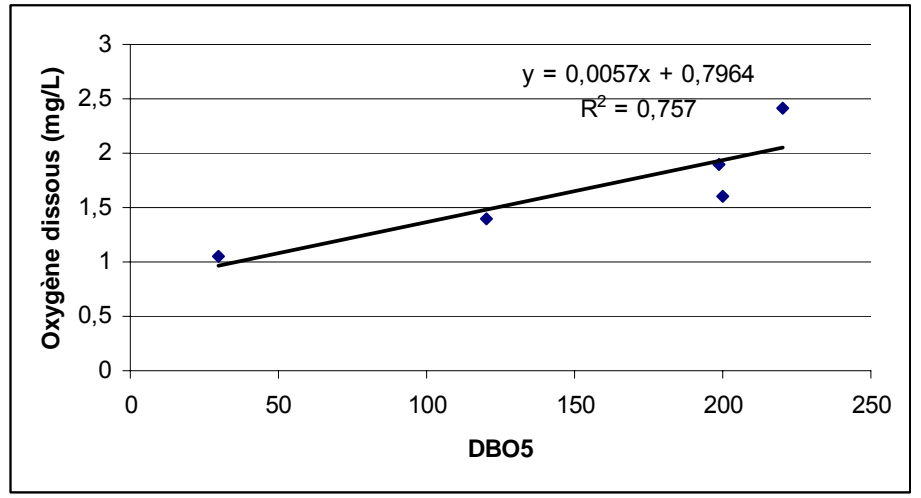

Figure 2: Corrélation établie entre l' $\mathrm{O}_{2}$ dissous et $\mathrm{DBO}_{5}$ des equx usées du rejet de l'abattoir de Kénitra (Maroc). 
Au niveau de ce rejet urbain, la charge organique est marquée par des valeurs très fortes de la $\mathrm{DBO}_{5}$, de la $\mathrm{DCO}$, des matières oxydables $(\mathrm{OM})$ et de faibles teneurs en oxygène dissous (Moyenne de $1,8 \mathrm{mg} / \mathrm{L}$ ). En fait, Ce rejet est le collecteur qui recueille la majorité des eaux usées domestique de la nouvelle Médina (population raccordée comprise entre 20.000 et 30.000 habitants) et draine les effluents de l'abattoir municipal et par conséquent, c'est le rejet le plus pollué. La pollution par les matières organiques, dégradables ou non, est essentiellement due aux rejets de l'abattoir municipal, d'une industrie d'engrais chimiques et aux rejets des populations urbaines raccordées à ce collecteur urbain.

\section{Conclusion}

Les eaux usées de l'abattoir municipal de la ville de Kénitra présentent des valeurs des paramètres physico-chimiques majeurs de pollution qui dépassent relativement les valeurs limites générales des rejets directs et indirects dans le milieu récepteur (estuaire de Sebou), ce qui représente un risque de pollution environnementale pour ce dernier d'où la nécessité d'un traitement de ces eaux usées brutes.

Au terme de l'évaluation de degré de pollution organique, on peut constater que l'ensemble des paramètres étudiés (en particulier avec la $\mathrm{DBO}_{5}$, la $\mathrm{DCO}$ et les MES) situent les eaux usées analysées dans la tranche a concentration moyenne à élevée [39]. Par ailleurs, selon la classification des effluents urbains réalisée par l'Office National de l'Eau Potable, ces eaux usées sont 5 à 7 fois plus chargées en matière organique que les eaux urbaines marocaines, [36]. En plus de la matière organique, elles contiennent les quantités adéquates d'azote organique pour subvenir aux besoins des microorganismes épurateurs des systèmes biologiques, cependant, les orthophosphates avec des concentrations faibles peuvent présenter un problème pour le traitement biologique [40]. On peut conclure que les eaux usées de ce rejet sont facilement biodégradable même si que les rapports $\mathrm{DBO}_{5} / \mathrm{DCO}$ et $\mathrm{MES} / \mathrm{DBO}_{5}$ sont élevés. L'examen du rapport $\mathrm{DCO} / \mathrm{DBO}_{5}$ souligne bien le caractère biodégradable des eaux usées de l'abattoir auxquelles un traitement biologique paraît tout à fait convenable.

Le traitement de ces eaux usées est nécessaire afin de produire un effluent qui respect les normes de rejets directs et indirects selon le Ministère de l'Environnement du Maroc [28]. L'abattoir de Kénitra génère une eau usée qui convient au traitement biologique en termes de DB05, MES, et substances nutritives (nitrates, nitrites et orthophosphates). Cependant, il faut tenir compte les caractéristiques physico-chimiques et microbiologiques des eaux usées domestiques mélangées avec les effluents de l'abattoir. 


\section{Remerciements}

Ce travail a été réalisé dans le cadre du projet PARS BIO N ${ }^{\circ}$ 148, Université Ibn Tofail, Faculté des Sciences de Kénitra. Maroc.

Nos vifs remerciements au Ministère de l'Education Nationale, de l'Enseignement Supérieur, de la Recherche Scientifique et de la Formation des Cadres. Rabat, Maroc.

\section{Références}

[1] - V. Johan et M.0. Mizier. Traitement des eaux industrielles. Effluents d'abattoirs : une pollution biodégradable. 2004 Eau, Industrie, Nuisances. Vol. 269. 33-43, ill,.

[2] - DA. Lovett, SM.Travers et KR. Davey. Activated sludge treatment of abattoir wastewater - I. Influence of sludge age and feeding pattern. 1984 Wat. Reș: 18: 429434.

[3] - D. Couillard et S. Gariepy. Faisabilité du procédé aérobie thermophile pour un effluent d'abattoir. 1990 Can. J. Chem. Eng. 68: 018-1023,.

[4] - R. Borja et MM. Duran. Influence of the support on the kinetics of anaerobic purification of slaughterhouse wastewater.1993. J. Biores. Technol. \# 44: 57-60..

[5] - D.I. Massé and L. Masse. Treatment of slaughterhouse wastewater in anaerobic sequencing batch reactors. 2000a Canadian Agricultural Engineering. Vol. 42, №. 3. 131-137..

[6] - E.H. Giglio Ponsano, P. Magalhaes Lacava and M.F. Pinto. Chemical composition of Rhodocyclus gelatinosus Biomass Produced in Poultry Slaughterhouse Wastewater. 2003 Brazilian Journal of Chemical Engineering. Vol. 46, № 02, pp. 143-147, March.

[7] - V. Reginatto, R.M. Teixeira, F. Pereira, W. Schmidell, A. Furigo, R. Menes, C. Etchebechere andH.M. Soqres. Anaerobic ammonium oxidation in a bioreactor treating slaughterhouse wastewater. Brazilian Journal of Chemical Engineering. Vol. 22, № 04, pp. 593-600, 0ctober-December 2003.

[8] - Gnagne et F. Brissaud. "Etude des limites de l'épuration par infiltration sur sable des eaux usées fortement chargées en matières oxydables de l'abattoir de Ougadougou au Burkina Faso II. Sud Sciences \& Technologies. N 9. Décembre 2002.

[9] - M. Asselin, J.-F. Blais, P. Drogui et H. Benmoussa. IUtilisation de l'électrocoagulation pour le traitement des eaux usées d'abattoir II. 21 e Congrés régional de l'est du Canada de l'Association Canadienne sur la Qualité de l'Eau. Québec, 4 novembre 2005. 
[10] - M.A. Bull, R.M. Sterritt and J.N. Lester. II The treatment of wastewaters from the meat industry: a review II. Environmental technology letters 3: 117-126. 1982.

[11] - G. Sachon. "Les eaux résiduaires des abattoirs de bétail". Gestion et Traitement. Tribune Cebedeau (1986) 515: 27-45.

[12] - SKI. Sayed. "Anaerobic treatment of slaughterhouse wastewater using the USAB process". phD thesis. 1987. Wageningen, The Netherlands: Agricultural University of Wageningen.

[13] - WP. Tritt et F. Shuchard "Materials flow and possibilities of treating liquid and solid wastes from slaughterhouse in Germany". 1992. Biores. Technol. \# 41: 235-245.

[14] - M.R. Johns. "Developments in wastewater treatment in the meat processing industry : A review. Bioresource technology 11 1995. 54: 203-216.

[15] - K.Q. Camin. "Cost of waste treatment in the meat packink industry". In proceeding of the 25th industrial waste conference, 193-202. 1970. Ann Arbour, mi: Ann Arbour Science.

[16] - J. Wéthé, M. Kientga, D. Koné et N. Kuéla. ॥ Profil du recyclage des eaux usées dans l'agriculture urbaine à Ouagadougou II. Visité d'Etude et Atelier International sur la réutilisation des eaux usées en agriculture urbaine: un défi pour les municipalités en Afrique de l'Ouest. Rapport final, 3-8 juin 2002 Ouagadougou - Burkina Faso. 183p.

[17] - B. Keraita. "Wastewater use in urban and peri-urban vegetable farming in Kumasi, Ghana". MSc. Thesis. Wageningen University, Wageningen, The Netherlands (2002).

[18] - T. Gnagne et F. Brissaud ॥ Etude des potentialités d'épuration d'effluents d'abattoir par infiltration sur sable en milieu tropical II. Sud Sciences \& Technologies. $N^{\circ} 11$. Décembre 2003.

[19] - H. Labaioui ॥ Biotransformation des déchets des abattoirs de la viande rouge et essai de valorisation du produit fermenté comme un stable bio-engrais (cas de Kénitra) II. Thèse Doctorat. Fac. Sci. Kénitra. Université lbn Tofail (2006). 240p.

[20] - Y. El Guamri "Contribution à l'évaluation de la qualité physico-chimique et parasitologique des eaux usées brutes de la ville de Kénitra (Maroc) II. Mémoire DESA. (2003). Fac. Sci. Kénitra. Univer sité Ibn Tofail. 149p.

[21] - ISO 5667/3. II Qualité de l'eau - échantillonnage - guide pour la conservation et la manipulation des échantillons 11 (1994).

[22] - DIN (Deutsche Industrie Normes). Détermination de la Demande Biologique en Oxygène (DBO) selon DIN Laboratoire National de I'Environnement - Rabat.. 1992a

[23] - (DCO) selon DIN 38409-H52. Laboratoire National de I'Environnement - Rabat.. 1992b. 
[24] - J. Rodier. II'analyse de l'eau naturelle, eau résiduaires, eau de mer II, 8 ème Edition, (1996) Dénod. Paris, 1383p

[25] - DIN (Deutsche Industrie Normes). Dosage des composés phosphoriques méthodes photométrique par phosphomolybdique (selon DIN 38405-D11-1 0-P043-). Laboratoire National de l'Environnement - Rabat. 1993a.

[26] - DIN (Deutsche Industrie Normes) 1993b. Dosage des nitrates par méthode photométrique avec 2,6 - diméthylphénol (selon DIN 38405-D9-2). Laboratoire National de l'Environnement - Rabat.

[27] - DIN (Deutsche Industrie Normes) 1993c. Dosage des nitrites par méthode photométrique (selon ISO 6777 resp en 2677. Laboratoire National de I'Environnement - Rabat.

[28] - Ministère de I'Environnement du Maroc, (2002). ॥ Normes marocaines, Bulletin officiel du Maroc $11, \mathrm{~N}^{\circ} 5062$ du 30 ramadan 1423. Rabat.

[29] - D.I. Massé and L. Masse. "ICharacterization of wastewater from hog slaughterhouses in Eastern Canada and evaluation of their in-plant wastewater treatment systemsII. 2000b. Canadian Agricultural Engineering. Vol. 42, №. 3. 139-146.

[30] - M. Chennaoui, 0. Assobhei et M. Mountadar. "Biostabilisation des eaux usées d'abattoir de la ville d'El Jadida (Maroc) II. Rev. Biol. Biotech. Vol.2, No 1, April 2002. pp. $44-48$

[31] - L.A.S Miranda., J.A.P. Henriques and L.O. Monteggia. "A full-scale uasb reactor for treatment of pig and cattle slaughterhouse wastewater with a high oil and grease content”. Brazilian Journal of Chemical Engineering. Vol. 22, № 04, pp. 601-610, 0ctobr-December, 2005.

[32] - J. Nisbet Verneaux. "Composantes chimiques des eaux courantes. Discussion et proposition de classes en tant que bases d'interprétation des analyses chimiques II. Ann Limnol (1970) ; 6: 161-9.

[33] - 0. Thomas. II Métrologie des eaux résiduaire II. Ed. Cebedoc / Tec. et Doc. 11, Liège -75384. (1995) Paris.

[34] - M. Khamar. II Etude de la charge en métaux lourds dans les eaux, sédiments, les sols et productions agricoles irriguées par les eaux uéses de la ville de Fès 11. Thèse de Doctorat (2002). Fac. Sci. Dhar El Mahraz. Fès. Maroc. 212p.

[35] - R. A Zerhouni. ॥ Flore algale des eaux usées de la ville de Fès et étude de la capacité de certaines espèces à éliminer la charge azotée, phosphatée et quelques métaux lourds (Chrome et Cadmium) II. Thèse de Doctorat. (2003) Fac. Sci. Dhar El Mahraz. Fès. Maroc. 146p. 
[36] - ONEP. Approche de la typologie des eaux usées urbaines au Maroc. ONEP et GTZ. Rabat (1998).

[37] H.W Pearson., D.D. Mara and C.R Bartone. "Guidelines for the minimum evaluation of the performance of full-scale waste stabilization pond system". 1987. Wat. Res. 21, $\mathrm{N}^{\circ}: 1067-75$.

[38] M. Henze, P. Harremoes, J. L.C Jansen. and E. "Avrin. Wastewater treatment". 2nd ed. (1997) springerverlag. Berlin.

[39] Metcalf et Eddy, INC. "Wastewater engineering: Traetment, Disposal and Reuse". 3ème Edition Library of Congress Cataloging in publication data. (1991) D. 645. T34.

[40] R. Hamdaoui. "Caractérisation physico-chimique et traitment des eaux usées résiduaires de la socièté SETEXAME. Kénitra II. Mémoire DESA. 2006. Fac. Sci. Kénitra. Université Ibn Tofail. 100p. 